\title{
Competencias Tecnológicas: Un Nuevo Modelo Pedagógico
}

\section{Technological Skills: A New Pedagogical Model}

Elibet Concepción Pérez y y Yasiel Reyes-Rodríguez ${ }^{2}$

\section{๑ EDICIÓN: \#-CIVTAC}

Recibido: 24/junio/2021

Aceptado: $18 /$ septiembre/2021

Publicado: 24/septiembre/2021

País

${ }^{1} \mathrm{Cuba}$

${ }^{2} \mathrm{Cuba}$

\section{IIIII Institución}

${ }^{1}$ Universidad de Artemisa

${ }^{2}$ Universidad de Artemisa

\section{Correo Eletrónico}

1perezely204@gmail.com

2superyasiel@gmail.com

\section{(iD ORCID}

${ }^{1}$ https://orcid.org/0000-0003-4856-0851 ${ }^{2} \mathrm{https}: / /$ orcid.org/0000-0002-3204-0926

\section{Citar así: C APA / IEEE}

Pérez, E. \& Reyes-Rodríguez, Y. (2021). Competencias Tecnológicas: Un Nuevo Modelo Pedagógico. Revista TecnológicaEducativa Docentes 2.0, 11(2), 76-83. https://doi.org/10.37843/rted.v11i2.246

E. Pérez y Y. Reyes-Rodríguez, "Competencias Tecnológicas: Un Nuevo Modelo Pedagógico", RTED, vol. 11, n. ${ }^{\circ}$, pp. 76-83, sep. 2021.

\section{Resumen}

En las últimas décadas del siglo pasado surgió un vertiginoso avance tecnológico en el mundo. Los sistemas educativos no quedaron alejados de estos avances, enseñar desde la virtualidad era un nuevo reto, esto provocó disímiles proyectos destinados a este fin. El desempeño docente en la práctica pedagógica requiere de un alto nivel de preparación y conocimiento por parte del profesor, es donde queda reflejado el trabajo metodológico e investigativo. La presente investigación tuvo como objetivo implementar un modelo pedagógico para mejorar el desempeño docente con enfoque de competencias tecnológicas. Se utilizó una metodología descriptiva para cada una de las cuatro etapas bajo un enfoque mixto. De los 52 profesores pertenecientes a la Universidad de Artemisa, se trabajó con 35, seleccionando una muestra no probabilística intencional. La consulta a especialistas se realizó a 7 profesores del Instituto Superior Pedagógico Rubén Martínez Villena, de ellos 5 con más de 25 años de experiencia en el nivel superior. Los resultados alcanzados sirvieron como punto de partida para caracterizar el desempeño docente con enfoque de competencias tecnológicas y sus indicadores a medir. Posteriormente, se les facilito a los profesores la construcción de sus propios recursos de aprendizaje relacionados con sus competencias identificadas, su contexto tecnológico y asignatura, esto hizo que el proceso docente resultara significativo.

Palabras clave: Modelo pedagógico, competencias tecnológicas, tecnología, docente.

\section{Abstract}

In the last decades of the previous century, a dizzying technological advance emerged in the world. The educational systems were not far from these advances; teaching from virtuality was a new challenge, which caused different projects for this purpose. Teaching performance in pedagogical practice requires a high level of preparation and knowledge on the part of the teacher, which is where the methodological and investigative work is reflected. The objective of this research was to implement a pedagogical model to improve teaching performance with a focus on technological competencies. A descriptive methodology was used for each of the four stages under a mixed approach. Of the 52 professors belonging to the University of Artemisa, 35 selected an intentional nonprobabilistic sample. The consultation with specialists was carried out with seven teachers from the Rubén Martínez Villena Higher Pedagogical Institute, 5 of them with more than 25 years of experience at the higher level. The results achieved served as a starting point to characterize teaching performance, focusing on technological competencies, and measuring indicators. Subsequently, it provided teachers with the construction of their learning resources related to their identified competencies, technical context, and subject; this made the teaching process significant.

Keywords: Pedagogical model, technological skills, technology, teacher. 


\section{Introducción}

En las últimas décadas del siglo pasado surgió un vertiginoso avance tecnológico en el mundo. Adell (1997) resalta que el sistema educativo no es precisamente un ambiente en el que la tecnología tenga un papel relevante para las tareas que allí se realizan, sus participantes se han mostrado reacios a incorporar novedades a su estilo de hacer las cosas. La sociedad de la información se convertirá en la sociedad del conocimiento y del aprendizaje. Los sistemas educativos no quedaron alejados de estos avances, enseñar desde la virtualidad era un nuevo reto, esto provocó disímiles proyectos destinados a este fin. Múltiples eran las promesas de esta nueva forma de educar, pero la revolución tecnológica en las instituciones educativas mantenía demasiadas deficiencias: los profesores no estaban a la altura de las exigencias de sus nuevos estudiantes (nativos digitales). La incorporación de las Tecnologías de la Informática y la Comunicación (TIC) para masificar su uso en los procesos docentes requiere un replanteo de los paradigmas educativos existentes hasta el momento; el desarrollo de habilidades específicas en cada individuo para facilitar su accesibilidad en este medio potenciando el proceso de enseñanza - aprendizaje.

En el decenio de los años 80 surge el enfoque de formación basado en competencias. Esto demuestra una tendencia creciente en utilizar las competencias en los procesos docentes, en los cuales desempeña un papel fundamental los profesores. Desde el punto de vista de Carrera \& Coiduras (2012) el desarrollo de competencias digitales del profesorado está, entre otros factores condicionado por su papel como formador y mediador en la adquisición que de ella hagan los estudiantes. Las universidades cubanas, particularmente la Universidad de Artemisa, no siguen el ritmo de esta tendencia dado que los profesores tradicionales, a pesar de contar con suficiente experiencia en el sector educacional, presentan dificultades en la educación digital, siendo necesaria una nueva forma de preparación y planificación de las actividades docentes.
Teniendo en cuenta a García (2017) el uso de las TIC promueve diversos cambios en el comportamiento de las personas o grupos sociales para que sean considerados miembros de los entornos digitales; sin embargo, para adquirir estas ventajas se requiere desarrollar habilidades importantes, tales como el diseño de una identidad digital que sea coherente con el uso de los diversos dispositivos y plataformas, con la finalidad de lograr una reputación en línea que sea favorable y lo consolide como fuente fiable. Para lograr esta nueva forma de preparación los autores consideran adoptar el enfoque de competencias en el proceso formativo a punto de partida de sus competencias tecnológicas, por lo tanto, existe una necesidad de desarrollar un modelo capaz de propiciarlo. Prendes \& Gutiérrez (2013) resalta que las TIC exigen que los docentes desempeñen nuevas funciones y también requieren nuevas pedagogías y nuevos planteamientos en la formación docente. Lograr la integración de las TIC en el aula dependerá de la capacidad de los maestros para estructurar el ambiente de aprendizaje de forma no tradicional,

Estos antecedentes sumados a vivencias acumuladas por los autores como docente con más de 10 años de experiencia le permitieron identificar como situaciones problemáticas: Limitada comprensión hacia una necesaria preparación tecnológica de los profesores vinculados al proceso docente. Poca preparación de los profesores para un desempeño docente con enfoque de competencias. Esto posibilitó identificar como contradicción fundamental: aquella que existe entre una necesidad de desarrollar el proceso formativo con enfoque de competencias tecnológicas en el proceso de enseñanza aprendizaje y una poca preparación de los profesores para un desempeño docente adecuado.

Esto hace necesario diseñar un modelo pedagógico para el mejoramiento del desempeño docente con enfoque de competencias tecnológicas de los profesores de la Universidad de Artemisa. Se manifiesta una significación práctica en el modelo propuesto, aplicando los instrumentos, los análisis, además del procesamiento estadístico, se pudo caracterizar el desempeño 
docente de los profesores, lo cual posibilitó un mejor desarrollo en el proceso docente. Es un modelo generalizable con fácil aplicación en otros contextos de formación de docente. $\mathrm{La}$ revolución digital ha impactado de forma decisiva en los más diversos ámbitos de la vida actual $\mathrm{y}$, por supuesto, también en el educativo: tanto en la forma de enseñar como en la de aprender. El concepto de aprendizaje ha dejado de ser un acto estático, efímero, definido por unos tiempos e instituciones determinadas, a ser una actividad constante, abierta y continua, sin tiempos ni espacios definidos (Adell \& Castañeda, 2016).

\section{Metodología}

González-Criollo (2018) indica que la práctica pedagógica es la razón de ser de la labor docente centrado en el proceso de aprendizaje y enseñanza; para efectivizarlo tiene que movilizar todos los elementos de la planificación (elementos, sujetos y procesos) para así obtener resultados que permitan a los educandos ponerse al nivel de su tiempo. Es por lo tanto que el desempeño docente constituye un factor importante en la calidad del aprendizaje, y este proceso se inicia con la toma de conciencia por parte del docente, debe propiciar la reflexión a fin de que sea capaz de autoevaluarse y sea consciente de cómo va en su labor diaria y si está obteniendo resultados favorables referente al rendimiento académico en los estudiantes.

Apoyándonos en investigaciones ya concretadas nos percatamos que era importante reconocer el estado actual del desempeño docente, competencias tecnológicas y habilidades que el profesor posee para elaborar estrategias que le permitan incorporar nuevas metodologías didácticas relacionadas con las TIC, para así lograr subsanar algunas dificultades que se encuentran presentes en el proceso enseñanza aprendizaje, lo que influye en desconocimiento teórico - práctico con que se manejan estas tecnologías en el contexto educativo, que por ende generan habilidades poco adecuadas por parte de los directivos y docentes, lo que no favorece a la docencia.

Teniendo en cuenta a Hernández, Hernández \& Baptista (2014), los enfoques cuantitativo, cualitativo y mixto constituyen posibles elecciones para enfrentar problemas de investigación y resultan igualmente valiosos. Son, hasta ahora, las mejores formas diseñadas por la humanidad para investigar y generar conocimientos. La investigación fue realizada con una metodología descriptiva obteniendo resultados cualitativos y cuantitativos por lo que tuvo un enfoque mixto.

Según Bisquerra (2004) un método es el camino para llegar a un fin. Los métodos de investigación constituyen el camino para llegar al conocimiento científico: son procedimientos o conjunto de procedimientos que sirven de instrumento para alcanzar los fines de la investigación. Para dar cumplimiento a ello se utilizaron como indagaciones teóricas se utilizó el método Histórico-Lógico que estuvo vinculado al estudio de los referentes, etapas del desarrollo histórico de desempeño docente con enfoque de competencias tecnológicas. Lo lógico nos permitió conocer las tendencias, cómo se ha desarrollado y aplicado este enfoque en el ámbito laboral además de su vinculación con el aspecto académico. El Sistémico estructural nos posibilitó interrelacionar, correlacionar los referentes teóricometodológicos del enfoque de competencias tecnológicas, analizar los instrumentos aplicados para diseñar el modelo propuesto

Como indagaciones empíricas fueron utilizadas: Revisión documental permitiendo identificar los criterios de los directivos y profesores sobre el comportamiento del desempeño docente con enfoque de competencias tecnológicas. En la encuesta a docentes se obtuvo información acerca del objeto de investigación, parte de ella constituyó su aplicación de un antes y un después de implementado el modelo. Consulta a especialistas fue utilizada con una finalidad de valorar la factibilidad, aplicación y viabilidad de la propuesta.

Otro elemento en el aspecto metodológico fue la selección realizada con la población objeto de estudio en la Universidad de Artemisa, Cuba. De los 52 profesores pertenecientes a la Universidad de Artemisa convocados para participar en dicha investigación, fueron escogidos 33 junto a sus 
dos directivos para un total de 35 participantes en la investigación, seleccionando una muestra no probabilística intencional. La consulta a especialistas aplicada a 7 profesores del Instituto Superior Pedagógico Rubén Martínez Villena, de ellos 5 con más de 25 años de experiencia en el nivel superior. En la actualidad el desempeño docente con enfoque de competencias constituye uno de los principales factores que influyen en la calidad del proceso docente educativo, motivo por el cual se hizo necesario caracterizar e identificar las competencias tecnológicas (Tabla 1) que debe poseer todo profesor o al menos un perfil básico de competencias tecnológicas.

\section{Tabla 1}

\section{Competencias identificadas en los profesores}

\begin{tabular}{|l|l|}
\hline Tecnológicas & $\begin{array}{l}\text { Conocimiento sobre TIC, destreza para navegar, comprensión, capacidad de } \\
\text { desenvolvimiento tecnológico, habilidades pedagógicas. }\end{array}$ \\
\hline Innovación - creatividad & $\begin{array}{l}\text { Capacidad para proponer soluciones novedosas diferentes a los métodos } \\
\text { tradicionales para aplicar a situaciones problemáticas del entorno. }\end{array}$ \\
\hline Pedagógicas & $\begin{array}{l}\text { Creatividad e innovación pedagógica, didáctica para aplicar las TIC, solucionar } \\
\text { problemas relacionados. }\end{array}$ \\
\hline Investigativa & Problematización sobre realidad educativa del docente frente a las TIC \\
\hline Actitudinales & $\begin{array}{l}\text { Disposición de los docentes para desarrollar el aprendizaje de los estudiantes. } \\
\text { Relación docente-conocimiento-estudiante. }\end{array}$ \\
\hline Evaluativas & Se conoce lo evaluado, los resultados obtenidos después de aplicadas las TIC. \\
\hline $\begin{array}{l}\text { Nota. } \text { La tabla presenta las Competencias identificadas en los profesores para lograr un mejor desempeño docente, } \\
\text { elaborada por los autores (2021). }\end{array}$
\end{tabular}

Posteriormente de identificadas las competencias se elaboraron los instrumentos para su aplicación, estos fueron validados por los especialistas ( 5 doctores en Ciencias y profesores titulares, 2 profesores auxiliares, máster en ciencias) lo cual involucró seleccionar variables a medir para obtener los valores identificativos mediante la interpretación estadística de los resultados recopilados de la muestra.

Revisión documental: Se confeccionaron guías para su realización, visitas a clases realizadas a los profesores para valorar su desempeño docente sobre superación e investigación relacionadas al enfoque de competencias tecnológicas. Entrevista a directivos estructurada, realizada a los 2 directivos del centro, un cuestionario a 33 profesores de varias asignaturas, de forma individual, previa información del objetivo antes de entrevista con una explicación del enfoque de competencias tecnológicas en el proceso formativo. Encuesta a docentes consistió en un cuestionario con preguntas cerradas y abiertas, aplicadas directamente por los investigadores.

\section{Resultados}

Hoy día las instituciones educativas deben, por un lado, hacer uso de la tecnología de la información en las clases y por otro, diseñar y poner en práctica planes de trabajo para incorporar y adquirir competencias. La incorporación de estándares TIC en la docencia significa la implementación de un cambio en las prácticas, una innovación en el quehacer profesional (Enlaces, 2009, p.111).

Alonso, Martínez, Castillo \& Muñoz (2015) observan que, en esta nueva era del conocimiento, el uso de las TIC en los procesos de enseñanza y aprendizaje ha originado cambios estratégicos en la educación, fomentando la existencia de modelos educativos que empleen o involucren su uso para favorecer el aprendizaje de los estudiantes a través de materiales educativos dentro de nuevos escenarios. Ello ha obligado a que cada día más los docentes requieran competencias que les permitan interactuar con los alumnos dentro de los procesos de formación, encontrando así nuevas formas para transmitir los diferentes saberes a éstos.

Cano (2017) resalta que la adquisición de competencias básicas y la inclusión de éstas en el currículo, a pesar de muchas resistencias a aceptarlas como tales, no está 
reñida en absoluto con la adquisición de contenidos. Al contrario: es total y necesariamente complementaria en la medida que algunas forman parte consustancial de los contenidos, mientras otras ayudan a adquirir y a afianzar los contenidos de cualquier área del currículo.

De la capacidad del profesor para integrar las TIC en el desarrollo curricular, depende que estos nuevos recursos didácticos cumplan sus tres funciones básicas: representar, presentar mundos reales y virtuales; facilitar al profesor la enseñanza; como, además, favorecer al alumno el aprendizaje. Funciones que son inseparables entre sí, respectivamente, en los tres elementos principales de los procesos de enseñanza-aprendizaje: los contenidos, los profesores y los alumnos. Area, Gutiérrez \& Vidal (2012, p.54).

Como afirma Buschman con gran profusión, que el mundo electrónico y ahora digital, ha trastornado cualquier noción unitaria de la alfabetización en sí misma debido a que estas tecnologías avanzadas la han "ampliado y al mismo tiempo fragmentado en muchas alfabetizaciones" (2014, p.26). La aplicación de las tecnologías a la formación universitaria debe superar la función de la transmisión y ser depositarios de información. Por el contrario, deben convertirse en herramientas que sean de verdad útiles para la creación de entornos diferentes para el aprendizaje y para la comunicación entre los participantes en la acción formativa (Cabero \& Llorente 2008, p. 12).

Las universidades cubanas, particularmente la Universidad de Artemisa, no siguen el ritmo de esta tendencia dado que los profesores tradicionales, a pesar de contar con suficiente experiencia en el sector educacional, presentan dificultades en la educación digital, siendo necesaria una nueva forma de preparación y planificación de las actividades docentes. Según Vásquez \& Eugenia (2013), ser competente, en otras palbras, saber hacer cosas o resolver situaciones, lo cual requiere saberes teóricos y prácticos, mucha imaginación y creatividad. Una educación transformadora con equidad implica la construcción de una sociedad competitiva en la que todos sus ciudadanos son empleables, lo cual se logra con todos sus recursos humanos capacitados (educados) en las competencias de la modernidad

Según Valle-Lima, plantea que los modelos resultan de utilidad por ser "una representación teórico-conceptual que permite aproximarse a un sistema o conjunto de fenómenos con la finalidad de facilitar su estudio, comprender sus procesos y eventualmente predecir su comportamiento" (2007, p.17). Los autores asumen que la propuesta es un modelo pedagógico que Valle-Lima concibe como un punto de partida para la transformación de la realidad que debe ser asumido también como un conjunto de acciones, distribuidas en etapas y enmarcadas en una estrategia de trabajo (2007, p. 11).

\section{Componentes estructurales del modelo}

El modelo se estructuro en dos niveles: conceptual y metodológico, lo que permitió el mejoramiento del desempeño docente con enfoque de competencias tecnológicas. En el nivel conceptual se evidencian los fundamentos que lo sustentan, ideas científicas, dimensiones y su finalidad. El metodológico, presenta su dinámica de aplicación en cuatro etapas diseñadas que abarcan un conjunto de acciones concretando su aplicación (ver Figura 1, Tabla 2):

Etapa I: Determinación de problemas y potencialidades

Etapa II: Planeación de las actividades para el mejoramiento del desempeño docente con enfoque de competencias tecnológicas.

Etapa III: Ejecución de las actividades.

Etapa IV: Evaluación del modelo pedagógico 


\section{Figura 1}

Modelo Pedagógico para el mejoramiento del desempeño docente con enfoque de competencias tecnológicas

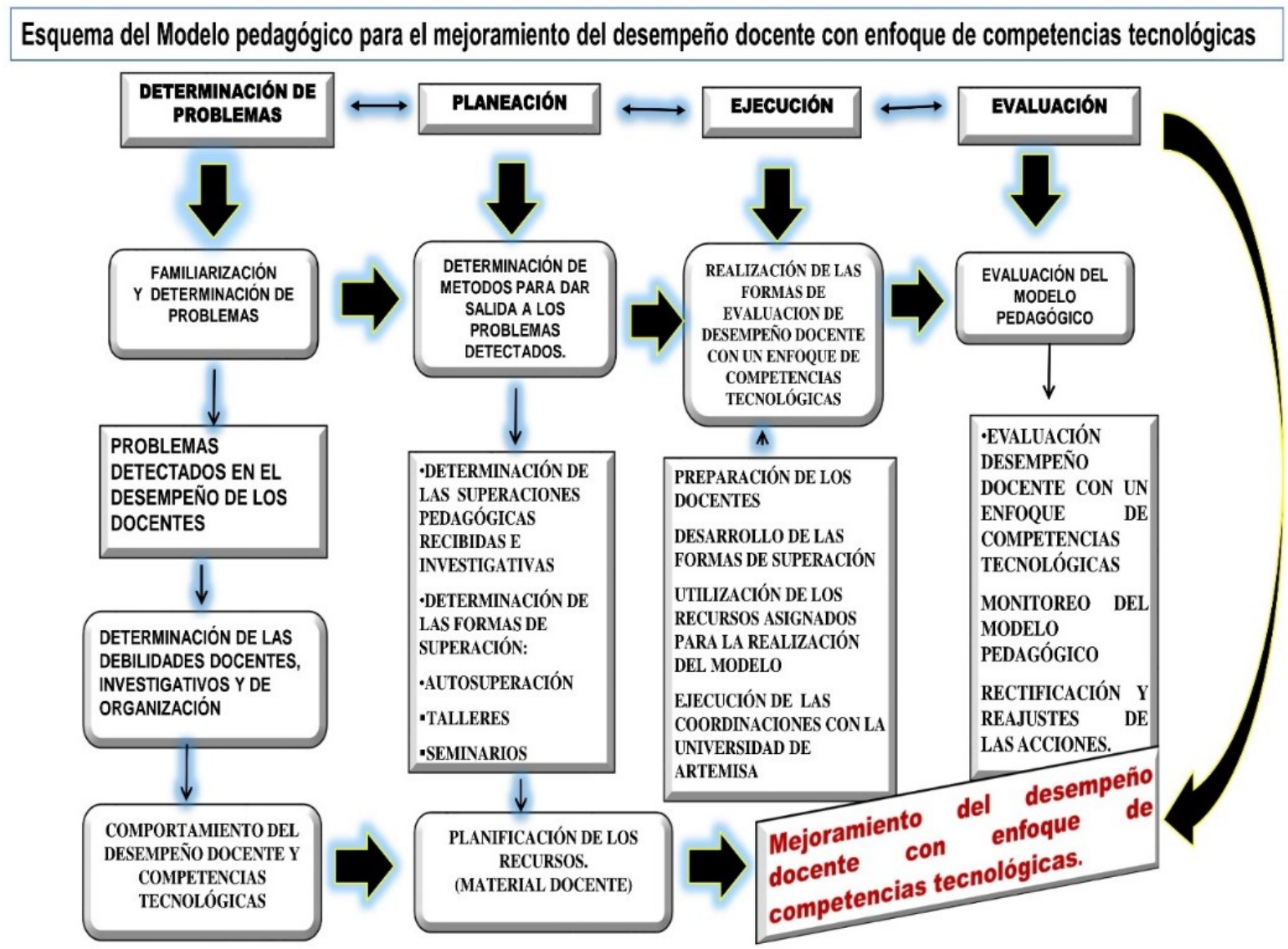

Nota. Es un modelo flexible que permite su adecuación, puede ser adaptado a las necesidades del contexto y proyecciones educativas, elaborado por los autores (2021).

\section{Tabla 2}

Evaluación de las competencias antes de aplicado el modelo pedagógico

\begin{tabular}{lllllll}
\hline \multirow{2}{*}{ Competencias } & Alto & \multicolumn{3}{c}{ Medio } & \multicolumn{3}{c}{ Bajo } \\
\cline { 2 - 7 } & Cant. & \% & Cant. & $\%$ & Cant. & $\%$ \\
\hline Tecnológica & 12 & 34.29 & 15 & 42.86 & 8 & 22.86 \\
\hline Innovación -creatividad & 23 & 65.71 & 12 & 34.29 & 0 & 0.00 \\
\hline Pedagógica & 8 & 22.86 & 23 & 65.71 & 4 & 11.43 \\
\hline Investigativa & 11 & 31.43 & 6 & 17.14 & 18 & 51.43 \\
\hline Actitudinales & 35 & 100.00 & 0 & 0.00 & 0 & 0.00 \\
\hline Evaluativas & 23 & 65.71 & 8 & 22.86 & 4 & 11.43 \\
\hline
\end{tabular}

Nota. La tabla presenta los resultados obtenidos de las competencias de los profesores antes de aplicado el modelo pedagógico, elaborada por los autores (2021).

En relación con las competencias consideras importantes por los docentes para introducir nuevas tecnologías en el aula, se identifican seis competencias. Los resultados en encuesta inicial muestran: en una muestra objeto de estudio de 35 profesores, solo 15 presentan un dominio medio en tecnología, esto representa el $42.8 \%$, lo cual evidencia una escasa preparación tecnológica de los docentes en el uso de las TIC en sus clases.
Otro aspecto importante para destacar radica en el $65.7 \%$ de los docentes encuestados, los cuales de acuerdo en la relación existente entre varias competencias como Innovación creatividad, evaluativas con el proceso evaluativo de los educandos además de los resultados obtenidos después de aplicadas las TIC, las cuales en su conjunto proporcionan una formación integral y una herramienta de 
trabajo tanto para el profesor como para el estudiante.

Resultando menos significativa la investigativa con un $31.4 \%$ así como un $22.8 \%$ en competencias pedagógicas, resultados relevantes pues estas competencias ayudan a viabilizar tanto conocimiento como habilidades partiendo de problemáticas identificadas por el docente. Sin embargo, el $100 \%$ de los profesores encuestados estuvo de acuerdo en competencias actitudinales, competencia fundamental en los docentes para desarrollar un proceso de enseñanza aprendizaje con calidad, aspecto de vital importancia para una posterior aplicación de metodologías capaces de incorporar TIC en su clase.

Lo antes expuesto indica la existencia de razones didáctico - pedagógicas por las cuales se debe incorporar nuevos modelos pedagógicos basados en competencias tecnológicas en los centros educativos, conllevando esto a incorporar TIC en cada clase. Esto se justifica en la posibilidad de las TIC tanto de ampliar como enriquecer el aprendizaje, dando paso al desarrollo de capacidades creativas, pensar independientemente, auto aprendizaje, entre otras (ver Figura 2).

\section{Figura 2}

Valoración de las competencias después de aplicado el modelo

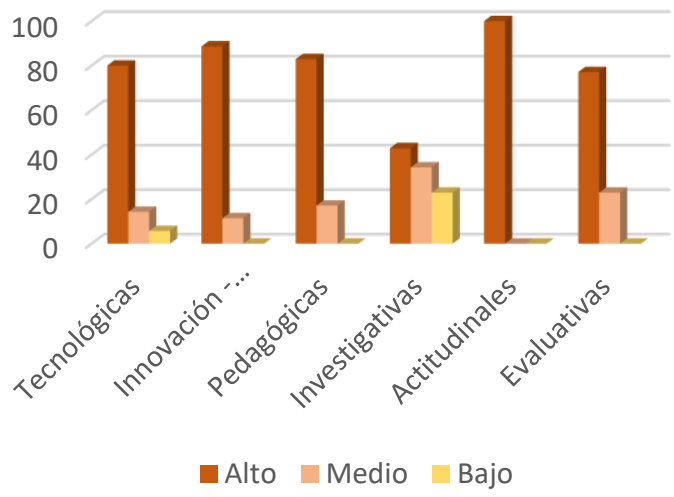

Nota. La gráfica presenta los resultados obtenidos de las competencias de los profesores después de aplicado el modelo pedagógico, elaborada por los autores (2021).

Los resultados obtenidos mediante una encuesta después de trabajar las actividades planificadas mostraron una calificación superior. En cada etapa puesta en práctica se valoró el $100 \%$ de competencias, donde se observa una media mayor en el dominio de competencias tecnológicas, así como su desempeño con un aumento considerable hasta el $80 \%$, aun quedando el $14.3 \%$ con un nivel medio en esta área. En cuanto al resto de competencias se muestra un alza en la media de valores con resultados positivos en el trabajo con TIC como una herramienta esencial en el proceso de enseñanza aprendizaje, resalta de Innovación creatividad con un nivel elevado de un $88.6 \%$.

El resto de las competencias se comportó de manera similar con un cambio significativo en competencias pedagógicas, esta subió a un $82.9 \%$. En el caso tanto de competencias Investigativas como Evaluativas el aumento no fue considerable, esto nos deja una puerta abierta a futuras actividades encaminadas a mejorar esta labor investigativa, así como la evaluativa, labores fundamentales en el trabajo de los docentes mediante una utilización de TIC. Resulta sumamente importante destacar la observación, después de aplicado el modelo en sus cuatro etapas planificadas, de un equivalente en nivel en competencias actitudinales manteniendo el $100 \%$ de los encuestados (muestra) este nivel alto. Se observó en ambos casos el mismo nivel de dominio en la variable estudiada. Corroborando así, lo relevante e importante de utilizar nuevos modelos pedagógicos basados en competencias tecnológicas para incorporar TIC en el proceso de enseñanza aprendizaje, logrando un desempeño docente elevado en los educandos.

\section{Conclusiones}

Una valoración positiva arrojada en la consulta de especialistas y los resultados del análisis comparativo aplicado antes y después de ejecutadas las actividades propuestas demuestran la viabilidad del modelo pedagógico elaborado por los autores. Posteriormente al trabajo con el modelo, se les facilitó a los profesores una construcción de sus propios recursos de aprendizajes, debidamente relacionados con su contexto tecnológico, su asignatura, su desempeño, lo cual hizo que el proceso resultara 
significativo. La alta motivación que genera el uso de los recursos tecnológicos en el entorno de las metodologías basadas en TIC promueve un aprendizaje mucho más significativo en los educandos. Cuando el profesor utiliza TIC como herramientas didáctico - metodológicas innovadoras genera cambios sustanciales en el interior del aula, lo cual favorece el proceso de enseñanza - aprendizaje de los estudiantes, además de enriquecer su desempeño docente. Aplicar el modelo pedagógico para el mejoramiento del desempeño docente con enfoque en competencias tecnológicas a todas las enseñanzas, proporcionaría una mayor calidad en el proceso de enseñanza aprendizaje del educando desde edades tempranas, contribuyendo a su preparación general para enfrentar situaciones problemáticas de la vida cotidiana.

\section{Referencias}

Adell, J. (1997). Tendencias en educación en la sociedad de las tecnologías de la información. EDUTEC, Revista Electrónica de Tecnología Educativa, 7.

Adell, J., \& Castañeda, L. (2016). Los entornos personales de aprendizaje (PLEs): Una nueva manera de entender el aprendizaje. In R. Roig Vila \& M. Fiorucci (Eds.), Claves para la investigación en innovación y calidad educativas. Alcoy: Marfil - Roma TRE Universita degli studi.

Alonso, M. Á., Martínez, V., Castillo, I., \& Muñoz, Y. (2015). Desarrollando Competencias Digitales en los Docentes. Pistas Educativas, 112, 439-459. http://www.itcelaya.edu.mx/ojs/index.php/pistas/arti cl e/viewFile/390/378

Area, M., Gutiérrez, A., \& Vidal, F. (2012). Alfabetización digital y competencias informacionales. Editorial Ariel.

Bisquerra, R. (2004). Metodología de la investigación educativa. La Muralla.

Buschman, J. (2014). Alfabetización informacional, «nuevas» alfabetizaciones y alfabetización. Boletín de la Asociación Andaluza de Bibliotecarios, 155183.

Cabero, J., \& Llorente, M. C. (2008). La Alfabetización Digital de los Alumnos. Competencias Digitales para el siglo XXI, 42(2), 7-28.

Cano, E. (2017). Las competencias de los docentes. En A. López Hernández (Ed.), El desarrollo de competencias docentes en la formación del profesorado. Ministerio de Educación y Ciencia.
Carrera, F. X., \& Coiduras, J. (2012). Identificación de la competencia digital del profesor universitario: Un estudio exploratorio en el ámbito de las ciencias sociales. Revista de Docencia Universitaria (REDU), 10(2), 273-298.

Enlaces. (2011). Competencias y estándares TIC para la profesión docente. Centro de Educación y Tecnología (Enlaces). Ministerio de Educación, Gobierno de Chile.

García, S. (2017). Alfabetización Digital Razón y Palabra, 21 (98) 66-8. http://www.redalyc.org/articulo.oa?id=1995531130 06

González-Criollo, J. (2018). Desempeño Docente, Facultad de Educación y Humanidades Programa Académico de Educación. Universidad Científica del Perú.

Hernández, R., Hernández, C. \& Baptista P. (2010). Metodología de la Investigación. McGraw Hill.

Prendes, P., \& Gutiérrez, I. (2013). Competencias tecnológicas del profesorado en las universidades españolas. Revista de Educación, (361), 196-222. https://doi.org/10.4438/1988- 592X-RE-2011-361140

Vásquez, H.\& Eugenia, L. (2013). Educación y modelos pedagógicos. Secretaría de Educación de Boyacá, Área Misional.

Valle-Lima, A. (2007). Metamodelos de la investigación pedagógica. Instituto central de ciencias pedagógicas. Ministerio de educación. Cuba. 